\title{
APLICABILIDADE DOS DIAGNÓSTICOS DE ENFERMAGEM COMO SUBSÍDIOS PARA INDICAÇÃO DO CATETER CENTRAL DE INSERÇÃO PERIFÉRICA
}

\author{
Applicability of nursing diagnosis as grants for indication of Central Catheter of Peripheral \\ Insertion \\ Aplicación de los diagnósticos de enfermería como subsidios para la indicación del catéter \\ central de inserción periférica
}

Leticia Celestino da Costa $^{1}$

Graciele Oroski Paes²

\section{RESUMO}

0 presente estudo teve como objetivo verificar a propriedade na aplicabilidade dos Diagnósticos de Enfermagem pelos enfermeiros como subsídio para a indicação do Cateter Central de Inserção Periférica (PICC). Trata-se de um estudo de abordagem descritiva, de tipologia mista, apropriando-se de preceitos quantitativos e qualitativos. A população-alvo constituiu-se de 10 enfermeiros devidamente habilitados à inserção do PICC, ativos e vinculados a um hospital estadual especializado. 0 instrumento aplicado constou de questionário com questões estruturadas e semiestruturadas devidamente escalonado com pontuações previamente definidas. De acordo com os achados, os enfermeiros apresentaram certa dificuldade para empregar os diagnósticos de enfermagem como parâmetro para indicação do PICC. Os enfermeiros habilitados recentemente apresentaram maior desenvoltura nas questões de associação dos fatores de risco e os diagnósticos de enfermagem. Portanto, são de grande relevância a educação continuada e a implementação do processo de enfermagem, visando o avanço teórico e prático da equipe.

Palavras-chave: Cateteres. Diagnóstico de enfermagem. Processos de enfermagem.

\begin{abstract}
The present study aimed to verify the property on the applicability of nursing diagnoses by nurses as a subsidy to the indication of Peripherally Inserted Central Catheter (PICC). This is a descriptive approach study, of mixed typology appropriating quantitative and qualitative ordinances. The target population consisted of 10 nurses duly qualified to the insertion of the PICC, active and linked to a specialized hospital of the State. The instrument used consisted of a questionnaire with structured and semi-structured properly scaled scores with scores previously defined. According to the findings the nurses had some difficulty to use nursing diagnoses as a parameter to indicate the PICC. The recently qualified nurses showed an ease regarding the issues related to the association of risk factors and nursing diagnosis. Therefore, it is very important the realization of continuing educations and the implementation of the nursing process in order to improve the team practical and theoretically.
\end{abstract}

Keywords: Catheters. Nursing Diagnosis. Nursing process.

\section{Resumen}

El presente estudio objetivó verificar la propiedad sobre la aplicabilidad de diagnósticos de enfermería por enfermeros como subsidio a la indicación del Catéter Central de Inserción Periférica (PICC). Enfoque descriptivo, de tipología mixta apropiándose de ordenanzas cuantitativa y cualitativa. La población objetiva fue de 10 enfermeros, autorizados para la inserción del PICC, activos y vinculados a un hospital estadual especializado. El instrumento aplicado consistió en un cuestionario con preguntas estructuradas con escala apropiada con puntuaciones anteriormente definidas. De acuerdo con los resultados los enfermeros tuvieron dificultad para usar diagnósticos de enfermería como parámetro para la indicación del PICC. Los enfermeros recién graduados tuvieron una mayor facilidad en los asuntos de asociación de factores de riesgo y diagnóstico de enfermería. Por tanto, es de gran importancia la realización de educación continua y la puesta en práctica del proceso de enfermería con el fin de promover el avance teórico y práctico del equipo.

Palabras clave: Catéteres. Diagnóstico de Enfermería. Procesos de Enfermería.

\footnotetext{
'Graduada em Enfermagem e Obstetrícia pela Escola de Enfermagem Anna Nery/ UFRJ.Rio de Janeiro - RJ. Brasil. E-mail: leleucacosta@hotmail.com; ${ }^{2}$ Doutora em Enfermagem pela Escola de Enfermagem Anna Nery. Prof. ${ }^{a}$ Assistente do Departamento de Enfermagem Fundamental/EEAN, vinculada ao Núcleo de Pesquisa de Fundamentos do Cuidado de Enfermagem/ NUCLEARTE. Rio de Janeiro - RJ. Brasil. E-mail: gracieleoroski@gmail.com
} 


\section{INTRODUÇÃO}

Entre os avanços tecnológicos e terapêuticos disponíveis para o cuidado aos clientes de terapia intensiva, ressalta-se o cateter central de inserção periférica (PICC, sigla em inglês correspondente a Peripherally Inserted Central Venous (atheter), que se encontra em expansão de uso pelos seus inúmeros benefícios, como a possibilidade da manutenção de uma terapia de duração prolongada com redução do número de punções, além de sua possibilidade de inserção à beira leito por enfermeiros, conferindo-lhes uma autonomia, no que se refere à escolha da melhor terapêutica ao cliente hospitalizado. A adequada qualificação e capacitação profissional conferem ao enfermeiro habilidades e conhecimentos necessários a indicação, inserção e manutenção do PICC, além de gerar um cuidado legitimado garantindo a autonomia profissional ${ }^{1,18}$.

Conforme a Lei $n^{0} 7498 / 86$ "é privativo ao enfermeiro o cuidado direto de enfermagem a pacientes graves, incluindo os cuidados de maior complexidade que exijam conhecimento científico, capacitação técnica e capacidade de tomar decisões 2". Sendo assim, observa-se que o enfermeiro possui legitimidade para a indicação do PICC, mas carece de autonomia para utilizá-lo na ausência do profissional médico. Isto remete uma reflexão acerca da autonomia do enfermeiro diante dos variados cenários e situações que tangenciam o ato de cuidar em sua dimensão técnica, organizacional e sistematizada, apropriando-se de fundamentações teórico-científicas e empíricas. Destarte, é sempre relativa, porque é construída no âmbito de diferentes relações que são lineares, envolvendo pessoas, instituições e as variáveis cotidianas.

De acordo com a resolução COFEN 358/09, a Sistematização da Assistência de Enfermagem (SAE), atividade privativa do enfermeiro, busca a identificação das situações saúde/doença dos indivíduos por meio da utilização de um método científico que irá subsidiar ações de enfermagem contribuindo para a promoção, prevenção, recuperação e reabilitação da saúde dos indivíduos. Ela é simplesmente a forma como o enfermeiro vem buscando agregar cientificidade para organizar as ações de prestação do cuidado. A utilização da SAE possibilita ao enfermeiro realizar com maior eficácia ações de supervisão, de avaliação e de gerenciamento dos cuidados prestados, bem como acompanhar os resultados das ações implementadas ${ }^{3}$. 0 Processo de Enfermagem (PE) deve estar baseado em um suporte teórico que oriente a coleta de dados, o estabelecimento de diagnósticos de enfermagem e 0 planejamento das ações ou intervenções de enfermagem ${ }^{4}$.

Esses aspectos dizem respeito aos elementos da prática profissional, por natureza, inseparavelmente ao Processo de Enfermagem: o que os agentes da enfermagem fazem (ações e intervenções de enfermagem), tendo como base o julgamento sobre necessidades humanas específicas (diagnóstico de enfermagem), para alcançar resultados pelos quais é legalmente responsável (resultados de enfermagem) ${ }^{5}$.
0 diagnóstico de enfermagem consiste no julgamento clínico sobre respostas dos indivíduos, família ou comunidades a problemas ou processos de saúde potenciais ou vitais, proporcionando uma base para a seleção de intervenções e obtenção de resultados finais que um enfermeiro planejou. Compreendido como uma ferramenta essencial para a aplicabilidade da SAE, possibilita individualizar o cuidado, transformar a prática da enfermagem, servir de base para as intervenções, organizar o saber da Enfermagem, introduzir o processo de enfermagem (método científico) na profissão, entre outras possibilidades. Tem como objetivo identificar os problemas existentes obtidos a partir da análise das informações coletadas na investigação e essenciais para um dos grandes valores do agir do enfermeiro: o planejamento do cuidado. ${ }^{6}$.

0 enfermeiro deve expor suas explicações e decisões para que assim, em consenso com a equipe multiprofissional, ambos possam optar pela melhor escolha aplicável a cada cliente. A autonomia profissional elucubra questões que perpassam por dimensões de cunho operacional e técnico, ante as discussões inerentes ao campo das percepções e autoimagem. 0 profissional enfermeiro reconhece a necessidade de se organizar para assumir uma dimensão maior no trabalho em saúde, por meio da implementação de estratégias para intervir de forma fundamentada no processo de cuidar?.

Vicejando uma interlocução entre as práticas organizacionais pela aplicação dos elementos do processo de enfermagem com as possibilidades decisórias para efetiva prescrição do PICC pelo enfermeiro, objetivamos verificar o conhecimento dos enfermeiros acerca dos diagnósticos de enfermagem aplicáveis à indicação e inserção do PICC e discutir as compatibilidades e não compatibilidades de respostas conforme os diagnósticos de enfermagem relacionados à sua inserção em adultos.

Deste modo, tal estudo justifica-se pelas evidências dos benefícios da utilização do PICC, que tem sido inserido mediante as indicações estritamente médicas, que, neste caso, são tão relevantes quanto às indicações da enfermagem. Estas por sua vez, são baseadas nos diagnósticos de enfermagem, oriundos da classificação dos sinais e sintomas observados pelos enfermeiros, e, assim, sua inserção corresponderia às intervenções cabíveis dos próprios diagnósticos.

\section{REVISÃO DE LITERATURA}

\section{Histórico da evolução do PICC}

No Brasil, o PICC passou a ser empregado a partir de 1990, primeiramente em neonatologia, devido ao seu pequeno diâmetro e à sua flexibilidade; seu uso em adultos teve início em 1995. É um dispositivo intravenoso inserido através de uma veia superficial da extremidade e que progride por meio de uma agulha introdutora e com a ajuda do fluxo sanguíneo, 
até o terço médio distal da veia cava superior ou da veia cava inferior, quando inserido pela veia safena, adquirindo características de cateter central. Esse dispositivo possui um ou dois lúmens. Quanto ao calibre, varia de 14 a 24 Gauge ou 1 a 5 French (Fr). É flexível, radiopaco, de paredes lisas e homogêneas, feito com material bioestável e biocompatível, como o silicone, poliuretano ou polietileno ${ }^{8,9}$.

As vantagens da utilização do CCIP, quando comparadas a outros cateteres centrais, estão bem documentadas: redução do risco de pneumotórax na inserção, redução do risco de sepse por colonização da pele em torno da inserção, reduzido custo na sua inserção quando comparados a outros cateteres tunelizados, menos desconforto relatado pelos pacientes e fácil manutenção ${ }^{10}$.

Por ser um dispositivo central, propicia maior hemodiluição da terapia IV administrada e redução dos riscos associados à infiltração, equimoses e hematomas e extravasamentos no subcutâneo, além de possibilitar uma segura assistência ambulatorial e/ou domiciliar, com maior relação custo/benefício ${ }^{11}$.

Dessa forma, inúmeros enfermeiros inserem o PICC, porém baseados em conhecimentos biomédicos. 0 estudo, porém, vem contrapor esta realidade, enfatizando autonomia do enfermeiro e o embasamento teóricoprático, oriundos do Processo de Enfermagem. Sendo assim, cabe à enfermagem desenvolver atividades para a manutenção e promoção da saúde, bem como para a prevenção de doenças, sendo de sua responsabilidade o diagnóstico e a intervenção de enfermagem. Segundo a literatura, nas décadas de 1920 e 1930, começaram a ser publicados estudos de caso envolvendo a análise e a avaliação sistemáticas de um cliente ou grupo de clientes similares, para promover a compreensão acerca da situação e das intervenções de enfermagem que se faziam necessárias ${ }^{12}$.

\section{Diagnósticos de enfermagem subsidiando as indicações do PICC}

0 termo diagnóstico de enfermagem tem sido utilizado em três principais contextos, como a segunda etapa do processo de enfermagem, como rótulo para uma lista de diagnóstico e como parte de uma afirmação sobre a resposta do indivíduo ao problema de saúde ${ }^{13}$. Cada diagnóstico aceito pela taxionomia da North American Nursing Diagnosis Association (NANDA), tem uma definição que busca explicitar a ideia ou o significado do nome do diagnóstico. Além da definição, cada diagnóstico de enfermagem é apresentado com uma listagem de características definidoras e outra de fatores relacionados ${ }^{14}$.

As características definidoras são as evidências que 0 enfermeiro identifica no paciente a partir do levantamento de dados e que pelo seu julgamento interpreta e agrupa. Os fatores relacionados são elementos que contribuem para a ocorrência do diagnóstico. Devem ser investigados, pois auxiliam na determinação das intervenções. Os diagnósticos podem ser atuais ou potenciais. Os potenciais, como se referem a situações de risco do paciente e não a respostas já apresentadas, não têm características definidoras ou fatores relacionados, mas têm fatores de risco que, tanto são a base para afirmá-los quanto os fatores que contribuem para o alto risco ${ }^{14,15}$.

A competência técnica e legal para o Enfermeiro inserir e manipular o PICC encontra-se amparada pela Lei 7498/86 e o seu Decreto 94406/87, além das Resoluções: COFEN n² 240/2000 (Código de Ética dos Profissionais de Enfermagem), Cap. III, das responsabilidades, nos seus artigos 16,17 e 18, COFEN n 258/2001, e, do parecer técnico COREN-RJ n0 09/2000, foi normalizada a inserção e a manipulação deste dispositivo pelo profissional enfermeiro ${ }^{16}$.

0 enfermeiro é reconhecido como profissional habilitado para a inserção do PICC, desde que seja submetido à qualificação e/ou capacitação profissional, o que irá lhe conferir os conhecimentos necessários a sua indicação, inserção e manutenção, além de medidas apropriadas de controle de complicações mecânicas e infecciosas relacionadas aos cateteres.

\section{MÉTODO}

Devido à frequente indicação e utilização do PICC em clientes adultos críticos, o cenário eleito foi uma instituição pública especializada em cardiologia, localizada no município do Rio de Janeiro (RJ).

Estudo com abordagem descritiva de tipologia quantitativa. Desenhos quantitativos descritivos retratam o que existe de fato, determinam a frequência em que este fato ocorre e categoriza a informação. 0 pesquisador observa, descreve e documenta vários aspectos do fenômeno. Não há manipulação de variáveis ou procura pela causa e efeito relacionados ao fenômeno ${ }^{17}$.

Como sujeitos da pesquisa, foram incluídos todos os enfermeiros ativos e habilitados à inserção do PICC da instituição, totalizando uma amostra não probabilística de dez enfermeiros. Os critérios de elegibilidade foram: ter habilitação para a inserção do PICC e ser ativo em gozo de suas atividades. Os critérios de exclusão foram estabelecidos mediante a recusa do profissional convidado em participar da pesquisa, mesmo após a leitura do Termo de Consentimento Livre e Esclarecido. A coleta de dados foi realizada durante os meses de novembro e dezembro de 2011.

Foi criado um instrumento de caráter original, composto por questões assertivas com resultados refutáveis e ratificáveis, sendo previamente submetido a dois pareceristas consultores ad hoc e doutores em enfermagem com especialidade na área de interesse. 0 arcabouço do instrumento foi estruturado em três partes: 
a primeira referente aos dados de identificação, formação acadêmica e profissional, tempo de habilitação e conhecimentos sobre os riscos e benefícios do PICC, além de prever aspectos sobre Sistematização da Assistência de Enfermagem e Diagnósticos de Enfermagem. A segunda parte compôs três questões objetivas, interpretativas e detalhadas sobre a indicação do PICC, fatores de risco e/ ou relacionados e diagnósticos de enfermagem. Por último, foi previsto um espaço para a análise e contribuições da pesquisa à luz dos colaboradores.

Aplicou-se o teste piloto a quatro enfermeiros, requerendo um remodelamento posterior ao teste. Em seguida, foi submetido a uma reanálise pelos pareceristas iniciais, sendo elencadas em destaque questões especificas sobre os benefícios do PICC, os possíveis fatores relacionados e/ou de risco que influenciam na escolha do cateter e a seleção dos diagnósticos de enfermagem corretos para fatores de risco específicos. Posteriormente, o instrumento redesenhado foi submetido aos dez enfermeiros, sujeitos da pesquisa. Neste grupo amostral, também estão inclusos os quatro enfermeiros participantes do piloto na análise. Sendo assim, todos os enfermeiros habilitados à inserção do PICC foram entrevistados.

Os dados obtidos foram tabulados e organizados em planilhas eletrônicas com representações gráficas (Microsoft Excel ${ }^{\circledR}$ ) e analisados utilizando o método de estatística descritiva, de frequência simples e percentual.

0 interesse em tela não pressupõe o tratamento dos resultados de enfermagem, mas sim ressaltar a aplicabilidade coerente e confiável dos Diagnósticos de Enfermagem como ferramentas garantidoras para a realização de prescrições de enfermagem efetivas e, portanto, para uma intervenção fundamentada e eficaz.

Este estudo está em conformidade com a Resolução 196/96 do Conselho Nacional de Saúde e foi submetida ao processo de aprovação do Comitê de Éticas em Pesquisa da instituição, sofrendo um parecer favorável de imediato, de processo de $n^{\circ} 2011 / 43$, na reunião de 23 de setembro de 2011, autorizado pelo Dr. Roberto Lauro Lana.

\section{RESULTADOS E DISCUSSÃO}

A Figura 1 (Gráfico 1 do Apêndice) faz referência às questões interpretativas, correlacionando os benefícios do PICC e o tempo de habilitação para inserção dos enfermeiros. Por meio dos achados, observou-se que, em relação aos benefícios quanto ao tempo de uso do cateter, redução do número de punções, características de uso e antibioticoterapia, a média de profissionais que acertaram as questões foi de 8 , dentre os quais os $75 \%$ ( $f a=6$ ) são habilitados de 1 a 5 anos e $25 \%$ $(f a=2)$ de 6 a 10 anos. 0 conhecimento destacado pelos enfermeiros caracteriza-se como eixo fundamental para difusão racional na utilização do PICC, proporcionando maiores benefícios e menores riscos de complicações. Sendo assim, 0 envolvimento do enfermeiro no que concerne à inserção e manutenção do PICC não o exclui da responsabilidade quanto aos riscos e benefícios, principalmente os riscos, para que, na tentativa de proporcionar a melhoria e manutenção da vida, não sejam causados malefícios à saúde.

Figura 1. Gráfico Benefícios do PICC $\times$ Tempo de habilitação para inserção do PICC.

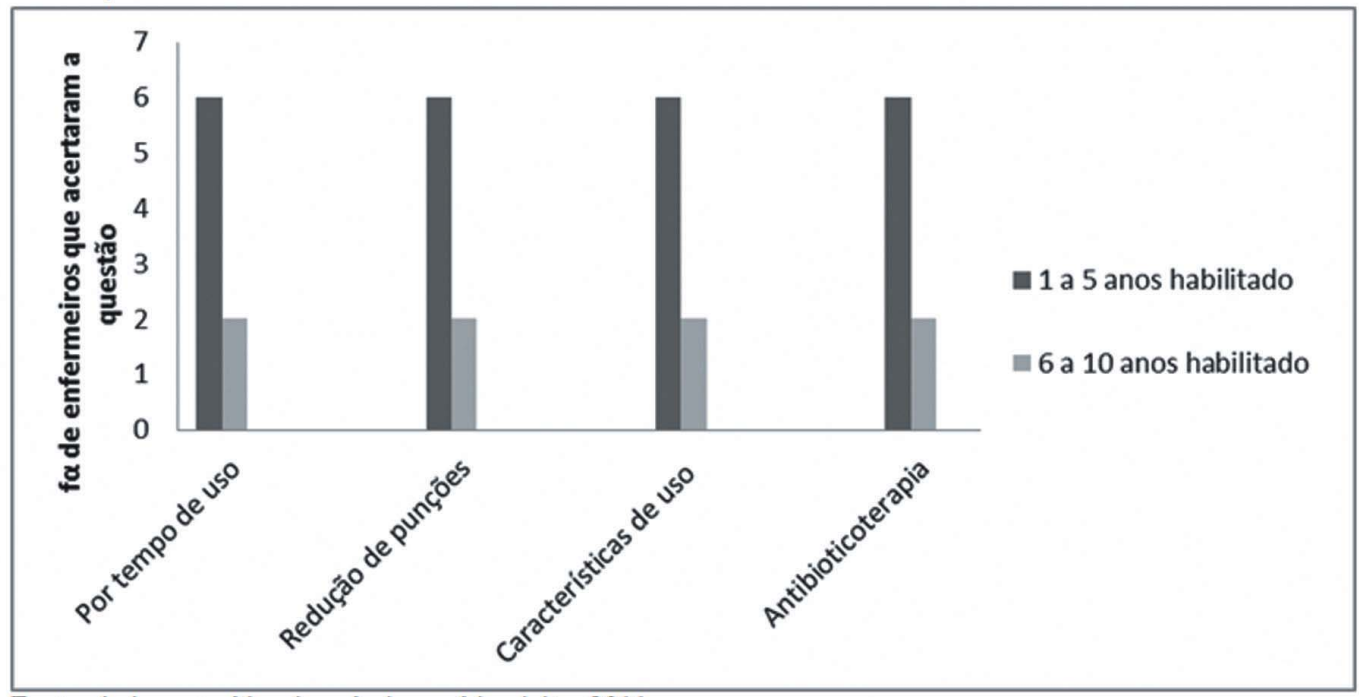

Fonte: dados extraídos do próprio cenário eleito, 2011 
A Figura 2 (Gráfico 2 do Apêndice) faz referência ao quantitativo de enfermeiros que acertaram a questão sobre os fatores de risco relacionados a indicação do PICC e o tempo de habilitação dos enfermeiros. Dos cinco fatores de risco citados corretamente no gráfico, os profissionais habilitados de 1 a 5 anos foram os que mais acertaram. Como exemplo, temos os fatores de risco imunossupressão e tempo de permanência, em que $100 \%$ dos profissionais que acertaram $\left(f_{\boldsymbol{a}}=3\right)$ encontravam-se neste período de habilitação.

Figura 2. Acertoss relacionados aos fatores de risco $\times$ Tempo de habilitação para a inserção do PICC

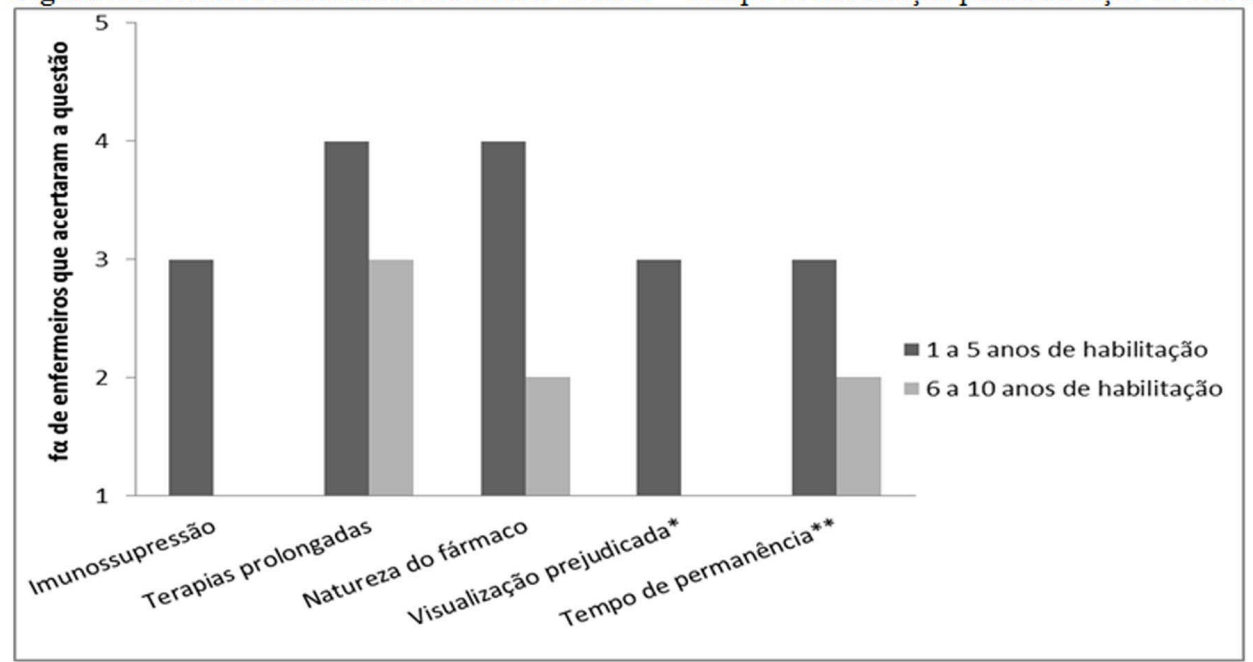

Fonte: dados extraídos do próprio cenário eleito, 2011.

* Referente ao fator de risco: Capacidade prejudicada de visualizar o local da inserção;

**Referente ao fator de risco: Duração do tempo de inserção do cateter;

A Figura 3 (Gráfico 3 do Apêndice) faz referência ao quantitativo de enfermeiros que acertaram a questão sobre fatores de risco relacionados à indicação do PICC e o tempo de habilitação para a inserção do PICC. Mesmo correspondendo ao grupo dos que mais acertaram a questão sobre os fatores de risco relacionados ao PICC, os profissionais habilitados de 1 a 5 anos também foram os que mais assinalaram fatores de risco errados relacionados a possíveis diagnósticos de enfermagem que influenciam na indicação do PICC. Os profissionais habilitados de 6 a 10 anos tiveram um quantitativo de erros menor, porém poucos souberam relacionar os fatores de risco com as indicações do PICC. Os fatores de risco mais assinalados erroneamente foram: sexo e idade, alteração da pressão arterial, imobilização e fraqueza muscular $(f a=1)$; arritmias ( $f a=4)$; edema; $80 \%(f a=4)$ encontravam-se no período de habilitação de 1 a 5 anos.

Apesar de pouco experientes, o conhecimento teórico dos enfermeiros habilitados até 5 anos é mais recente, facilitando o desempenho na abordagem metodológica e desenho do instrumento com questões teórico-práticas. Cabe aos profissionais mais experientes 0 aprimoramento contínuo com vistas à renovação e ampliação de conhecimento técnicocientífico aplicado à realidade da prática assistencial.

Figura 3. Erros relacionados aos fatores de risco $\times$ Tempo de habilitação para a inserção do PICC.

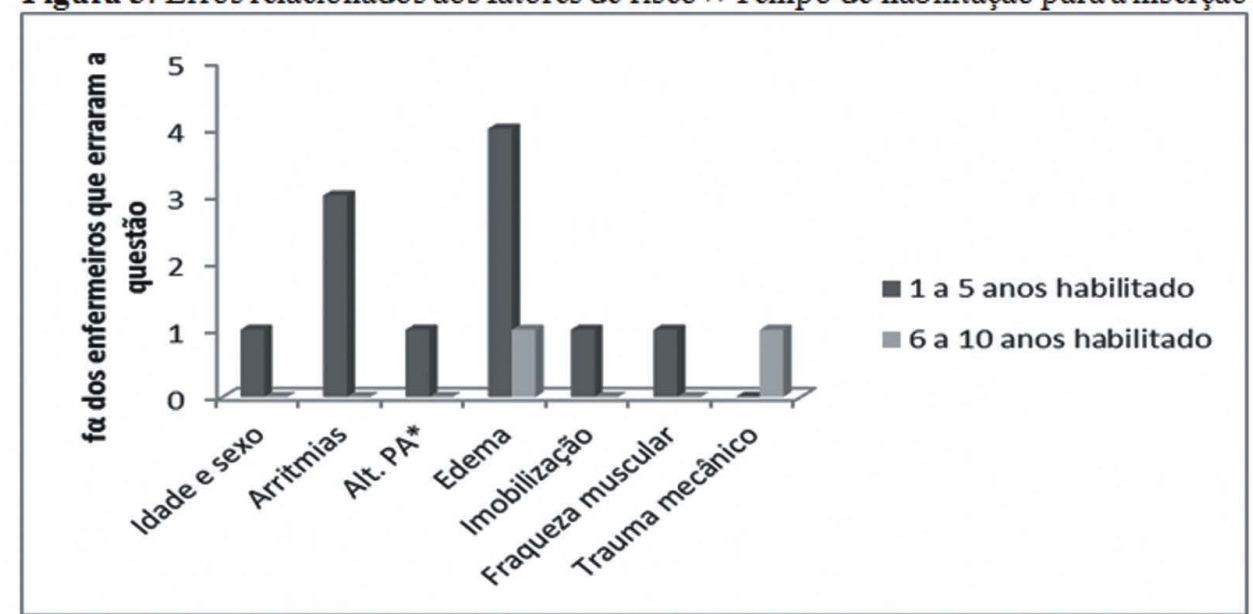

Fonte: dados extraídos do próprio cenário eleito, 2011.

*Referente ao fator de risco: Alteração da pressão arterial fora dos parâmetros aceitáveis; 
A Figura 4 (Gráfico 4 do Apêndice) faz referência aos acertos sobre os diagnósticos de enfermagem na utilização do PICC e ao tempo de formação dos enfermeiros. A análise do gráfico evidencia um resultado similar, em termos acertativos, dos enfermeiros formados nos três períodos citados. Isso pode ser observado nos diagnósticos risco de lesão e integridade da pele prejudicada, em que 33,33\% ( $f \boldsymbol{a}=2$ ) de cada período de formação acertaram.

Figura 4. Acertos relacionados aos Diagnósticos de Enfermagem $\times$ Tempo de formação.

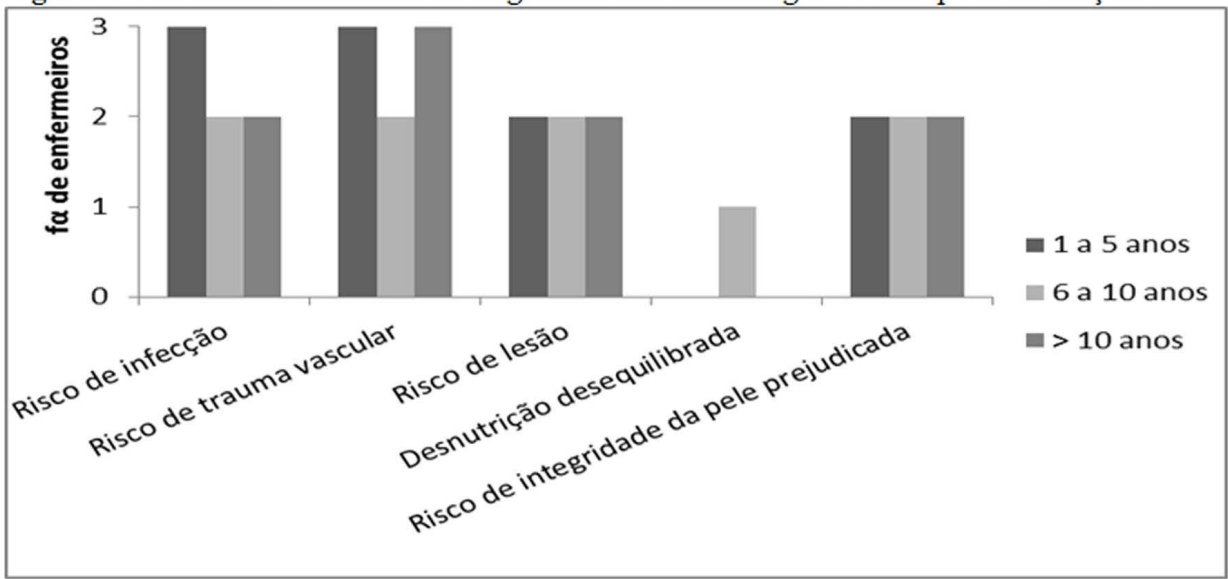

Fonte: dados extraídos do próprio cenário eleito, 2011.

A Figura 5 (Gráfico 5 do Apêndice) faz referência ao erros sobre os diagnósticos de enfermagem na utilização do PICC e ao tempo de formação dos enfermeiros. Dos enfermeiros que marcacam erroneamente o diagnóstico mobilidade no leito prejudicada, $50 \%(f a=2)$ foram os formados de 1 a 5 anos. Os enfermeiros formados há mais de 10 anos corresponderam a $100 \%(f a=1)$ dos que assinalaram o diagnóstico de débito cardíaco diminuído e corresponderam a $80 \%(f a=4)$ dos que assinalaram o diagnóstico de integridade da pele prejudicada.

Figura 5. Erros relacionados aos Diagnósticos de Enfermagem $\times$ Tempo de formação.

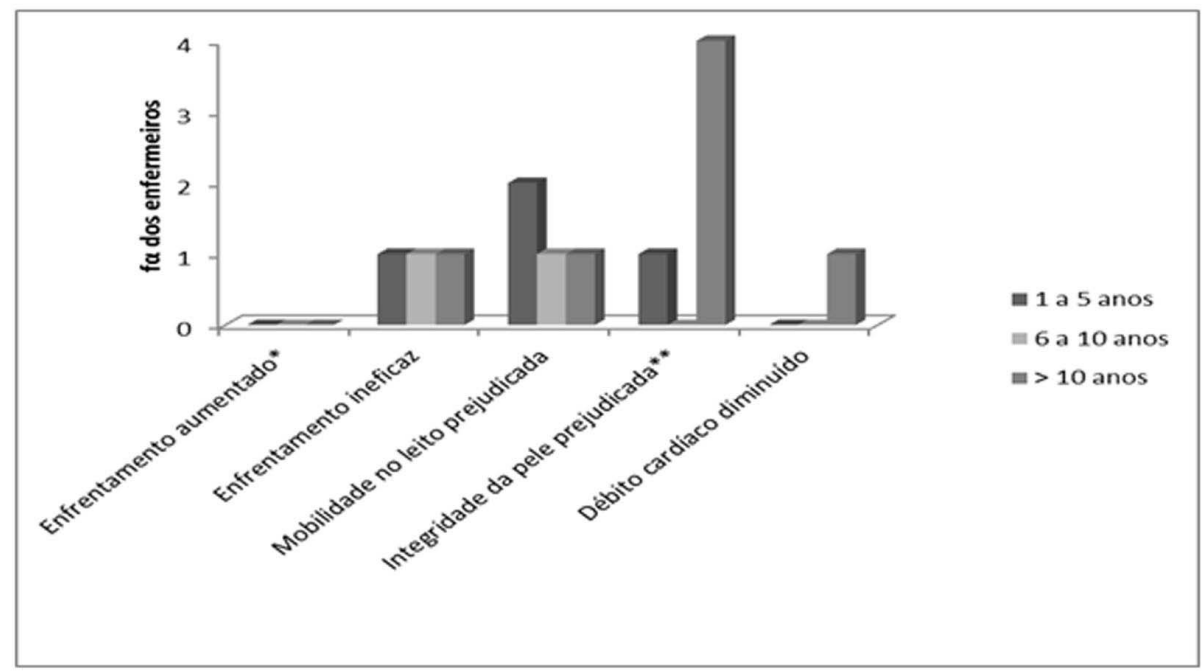

Fonte: dados extraídos do próprio cenário eleito. 2011

*Refere-se ao diagnóstico de enfermagem: Disposição para o enfrentamento aumentada;

**Refere-se ao diagnóstico de enfermagem: Integridade da pele prejudicada: menos que as necessidades corporais.

Com esse resultado observa-se a inter-relação entre a ênfase dada aos diagnósticos de enfermagem nas graduações nos últimos anos e a experiência profissional, oriunda do tempo de serviço. 0 grupo formado há mais tempo teve maior dificuldade em diferenciar os diagnósticos e enquadrá-los corretamente por ter sido um assunto menos explorado à época, diferentemente da formação contemporânea, em que o processo de enfermagem e a linguagem diagnóstica são temas de grande abordagem e relevância ligados ao planejamento do cuidado e agentes garantidores da autonomia profissional significativamente legitimada ${ }^{18}$.

0 domínio dos Diagnósticos de Enfermagem e seus respectivos fatores relacionados e/ou de risco são essenciais para uma classificação de excelência e um cuidado 
sistematizado. Quando empregados apropriadamente, os Diagnósticos de Enfermagem são considerados ferramentas imprescindíveis na formulação das prescrições de enfermagem, delineando pontualmente as atribuições e responsabilidade da equipe. Da mesma forma, a inserção do PICC demanda diagnósticos bem suportados cientificamente para que as prescrições de enfermagem sejam efetivamente empregadas. 0 "olhar" meticuloso do enfermeiro atento aos determinantes clínicos e científicos asseguram um bom diagnóstico, por seguinte minimiza a possibilidade de riscos para a segurança e o confor to do cliente.

Para tanto, a busca incessante pela reafirmação da autonomia profissional perpassa pela incorporação do processo de enfermagem aplicado a SAE, bem como pela avaliação do seu impacto tanto para a clientela quanto para os cenários de prática assistencial, educacional e científica ${ }^{1,12}$.

A "autonomia legitimada" significa para o enfermeiro o reconhecimento, pelo instituído e instituinte, das forças científicas e singulares do cuidar em enfermagem. A partir de incorporações de saberem próprios e alheios a sua prática, imprime um olhar de vanguarda no que tange à construção de um saber fazer único e singular à enfermagem. Isso posto, quando incorporados como processo de enfermagem aplicado, geram autonomia e legitimam a identidade do enfermeiro ${ }^{18}$.

\section{CONCLUSÃO}

Por meio deste estudo sobre as indicações do PICC pelos Diagnósticos de Enfermagem, conclui-se que os objetivos de verificar o conhecimento dos enfermeiros acerca dos diagnósticos de enfermagem aplicáveis à indicação e inserção do PICC e discutir as compatibilidades e não compatibilidades de respostas conforme os diagnósticos de enfermagem relacionados à sua inserção em adultos foram alcançados.

Em relação ao conhecimento dos diagnósticos e o tempo de habilitação, pode-se dizer que este é um fator muito importante quando se refere ao planejamento e intervenções de enfermagem. Os enfermeiros habilitados mais recentemente apresentaram maior desenvoltura quanto às questões de associação dos fatores de risco e os diagnósticos de enfermagem. Isso foi notório na apresentação dos resultados quanto ao tempo de formação profissional, ao tempo de habilitação e à propriedade de trabalhar diagnósticos de enfermagem empregados neste contexto. Portanto, são de grande relevância a realização de educações continuadas com a equipe de enfermagem e a implementação do processo de enfermagem, visando a avanço do conhecimento teórico e prático da classe pesquisada.

Por se tratar de um estudo inicial, desejamos, como contribuição futura, propor a realização de novos estudos como este. Torna-se mister a exploração da temática dos diagnósticos nas continuidades de pesquisa, em especial nas pesquisas de cunho transdirecionais, ou seja, com aplicabilidade à prática do cotidiano do enfermeiro, à utilização da Classificação de Intervenções de Enfermagem (NIC) e Classificação dos Resultados de Enfermagem (NOC), que enfatiza o uso de uma linguagem clara e útil, capaz de avaliar os cuidados por meio do emprego dos resultados de enfermagem.

\section{REFERÊNCIAS}

1. Paes GO, Leite IL, Melo ECP. Caring for the client with acute respiratory disorders: a proposed protocol for assistance to make decisions in nursing. Online Braz J Nurs. 2011; 10(1). Disponível em: http:// www.objnursing.uff.br/index.php/nursing/article/view/j.16764285.2011.3219.1

2. Decreto-lei n 94.406, de 08 de junho de 1987. Regulamenta a Lei ${ }^{0}$ 7498/86 sobre o exercício da enfermagem e dá outras providências. Diário Oficial da República Federativa do Brasil, Brasília, DF. jun 1987: 8853-855.

3. Torres E, Christovam BP, Fuly PCS, Silvino ZR, Andrade M. Sistematização da assistência de enfermagem como ferramenta da gerência do cuidado: estudo de caso. Esc Anna Nery. 2011; 15(4): 730-36.

4. Conselho Federal de Enfermagem (BR). Resolução n ${ }^{\circ}$ 358, de 15 de outubro de 2009. Sistematização da assistência de enfermagem e a implementação do processo de enfermagem. Diário Oficial da Republica Federativa do Brasil, Brasilia,DF, 20 out 2011; Seção 1.

5. Sabino B. Inserção e manutenção de PICC em UTIN: a necessidade de uma assistência de enfermagem peculiar.[on-line] [citado 2011 jan] Disponível em: www.webartigos.com/artigos/insercao-e-manutencao-depicc-em-utin-a-necessidade-de-uma-assistencia-de-enfermagem-peculiar/ $57125 /$

6. Kruse MHL, Silva KS, Ribeiro RG, Fortes CV. Ordem como tarefa: a construção dos diagnósticos de enfermagem. Rev Bras Enferm. 2008 mar/abr; 61(2): 262-66.

7. Bueno FMG, Queiroz MS. 0 enfermeiro e a construção da autonomia profissional no processo de cuidar. Rev Bras Enferm. 2005 mar/abr; 59(2): 222-27.

8. Jesus VC, Secoli, SR. Complicações acerca do Cateter Venoso Central de Inserção Periférica- PICC. Cienc Cuid Saude. 2007 abr/jun; 6(2): 252-60. Disponível em: http://www.periodicos.uem.br/ojs/index.php/ CiencCuidSaude/article/view/4174/2762

9. Pezzi M0, et al. Manual de Cateterização Central de Inserção Periférica CCIP/PICC. Grupo de estudos do CCIP. Porto Alegre: Associação Hospitalar Moinhos de Vento; 2004.

10. Di Giacomo M. Comparison of three peripherally-inserted central catheters: pilot study. British J Nurs. 2009; 18(1): 8-16. 
11. Volkmer A, Maestri R. I Curso de qualificação em utilização, inserção, manutenção e cuidados com o Cateter Central de Inserção PeriféricaPICC/CCIP adulto, pediátrico e neonatal do Instituto de Educação e Pesquisa do Hospital Moinhos de Vento. Porto Alegre; 2008.

12. Nascimento KC, Backes DS, Koerich MS, Erdmann AL. Sistematização da assistência de enfermagem: vislumbrando um cuidado interativo, complementar e multiprofissional. Rev Esc Enferm USP. 2008; 42(4): 643-48.

13. Carpenito ᄂ. Diagnóstico de enfermagem. $8^{\mathrm{a}}$ ed. Porto Alegre: Artes Médica; 2001.

14. Pasini D, Alvim I, Kanda L, Mendes RSP, Cruz DALM. Diagnósticos de enfermagem de pacientes internados em Unidades de Terapia IntensivaUTI. Rev Esc Enferm USP. 1996 dez; 30(3): 501-18.

15. Camargo PP, Kimura AF, Toma E, Tsunechiro MA. Localização inicial da ponta de cateter central de inserção periférica em recém-nascidos. Rev Esc Enferm USP. 2008 dez; 42(4): 45-54. Disponível em: http:// bases.bireme.br/cgi-bin/wxislind.exe/iah/online/;

16. Peixoto GMD, Mendonça AEO, Dantas RAN, Dantas DV, Torres GV. Tendências da produção científica sobre o cuidado de enfermagem no uso do cateter venoso central de inserção periférica. Rev Enferm UFPE. 2011 maio; 5(3): 806-11.

17. Sousa VD, Driessnack M, Mendes ICM. Revisão dos desenhos de pesquisa relevantes para enfermagem. Parte 1: desenhos de pesquisa quantitativa Rev Latino-Am Enfermagem. 2007 maio/jun: 15(3): 502-07

18. Paes GO. Gerenciando o cuidado de enfermagem com protocolos assistenciais: a práxis em enfermagem e sua interface com a tecnologia em saúde [tese]. Rio de Janeiro: Escola de Enfermagem Anna Nery, Universidade Federal do Rio de Janeiro; 2011. 